$\xi_{p}$

\title{
Noise Level Based Denoising Technique Utilizing Patch- Based Noise Level Estimator for Low-Light Condition Surveillance Image
}

\author{
Suhaila Sari ${ }^{1 *}$, Wong Zhi Lin ${ }^{1}$, Hazli Roslan ${ }^{2}$, Nik Shahidah Afifi Mohd Taujuddin ${ }^{1}$, Chua King Lee ${ }^{1}$, \\ Siti Zarina Mohd Muji ${ }^{1}$. \\ ${ }^{1}$ Faculty of Electrical and Electronic Engineering, Universiti Tun Hussein Onn Malaysia, Johor, Malaysia. \\ ${ }^{2}$ Faculty of Engineering Technology, Universiti Tun Hussein Onn Malaysia, Johor, Malaysia. \\ *Corresponding authors E-mail: suhailas @uthm.edu.my
}

\begin{abstract}
Digital Image Processing is a method to obtain image or to take out useful details or feature from image. The noise will cause the results of error in the image acquisition process. Generation of higher noise levels in the low light condition environment will often result in oversmoothed edges and textures during the denoising process because of lower signal levels in the image. Thus, this study goal is to improve denoising techniques for Poisson noise removal in low light condition for surveillance images. The Patch-Based Noise Level Estimator is designed to estimate the noise level of noisy image. The noisy image then fed to either OTSU WIE-WATH Filter or OTSU KU-WIE-WATH Filter automatically based on the noise level of image. The OTSU WIE-WATH Filter is used for low and medium Poisson noise removal while OTSU KU-WIE-WATH Filter is used mainly for high Poisson noise removal. The proposed denoising technique performances are analyzed with other existing denoising techniques in terms of Mean Absolute Error (MAE), computational time and visual effect inspection. The results verified that proposed technique is effective in removing different level Poisson noise in low light condition surveillance images.
\end{abstract}

Keywords: Denoising; Low-Light Condition; Mean Absolute Error; Poisson noise; Surveillance image acquisition.

\section{Introduction}

Digital image processing techniques help in manipulation of the digital images by using computers. All data types should undergo three common phases while using digital technique which are preprocessing, enhancement, and display, information extraction to improve the pictorial information for human interpretation [1].

The term surveillance means the observation or monitoring of a person, object or place intentionally. The definition to include "placee means that cameras installed to observe an open area, such as an outdoor mall or park, would constitute surveillance. One of the general surveillance devices closed-circuit television (CCTV) system are now networked and image can be stored, searched, analyzed, reproduced and made available on the internet. Digital cameras are still limited in taking images in low-light conditions. Smaller sensors in the camera resulting in generation of higher noise levels [2]. This will often result in oversmoothed edges and textures during the denoising process because of lower signal levels in the image. Poisson noise is cause when number of photon sensed by the sensor is not sufficient to provide detectable statistical information. Magnitude of the noise increases with the intensity of light [3].

Noise removal is one of the essential processes of analysing an image in digital image processing. The generation of noises in the image reduces the quality of the images. In common, there are two types of image denoising model, linear model and non-linear model. Generally, linear model (e.g. Mean Filter and Wiener Filter) are commonly used for image denoising. The advantage of linear filter is the speed. On the other hand, the models usually unable to preserve edges of the images in an efficiently. However, non-linear models (e.g. Bilateral Filter, Median Filter, Maximum Filter and Minimum Filter) are known to be effective in preserving edges in a much better way than linear models but slower since they are usually computationally expensive [3]. OTSU WIEWATH Filter and OTSU KU-WIE-WATH Filter [4] that proposed recently also improve the performance of eliminated the Poisson noise in an effective way. The filter allows the noisy pixels in the dark regions to be removed while preserving the texture regions to avoid over smoothing of test images.

\section{Literature Review}

\subsection{Existing Noise Estimation Techniques}

Estimation of image noise variance in [5] proposed a novel algorithm for estimating the noise variance of an image. The image is assumed to be corrupted by Gaussian distributed noise. The estimation of the noise variance is performed in three steps. First, the noisy image is filtered by a horizontal and a vertical difference operator to suppress the influence of the original image. The second step involved computation of a histogram of local signal variances. The desired estimation value is provided by a statistical evaluation of the histogram at the last step. A total of 128 natural and artificial test images is used for comparison with previously published estimation methods. It is observed that the novel algorithm provides more accurate results. However, the main difficulty 
of filter-based method is that the assumption of the difference of the two images to be noise but it is not always true, especially for images with complicated structures or fine details.

Block-Based Noise Estimation Using Adaptive Gaussian Filter [6] proposed a fast noise estimation algorithm using a Gaussian filter. It is based on block-based noise estimation, where an input image is assumed to be corrupted by the additive white Gaussian noise and an adaptive Gaussian filter is used in the filtering process. Functions of the standard deviation of the Gaussian noise are the coefficients of a Gaussian filter that is estimated from an input noisy image. An image is split into a number of blocks and smooth blocks that are classified by the standard deviation of a block's intensity are chosen in the process of amount of noise estimation (i.e., standard deviation of the Gaussian noise). The difference of the selected block images between the noisy input image and its filtered image is the standard deviation. The performance of the proposed method is compared with the other three conventional (block-based and filtering-based) noise estimation methods. The noise estimation algorithm is suitable to be applied for noise reduction in image and video applications such as digital cameras and digital television (DTV) due its performance and simplicity. Although the pro-posed algorithm is simple yet effective, it may overestimate the noise level for small noise level cases and might underestimate the noise level in large noise level cases. This is due to the variation of patch selection result based on the input image and noise level.

Single-Image Noise Level Estimation for Blind Denoising [7] proposed a patch-based noise level estimation method is introduced and suggested so that the noise level parameter can be tuned based on the complexity of the scene. This paper includes the selection process of low-rank patches without high frequency components from a single noisy image. The selection is made depending on the gradients of the patches and their statistics. Then, the noise level is estimated from the selected patches. This method can accurately estimate the addictive white Gaussian noise level of images without homogeneous areas. How-ever, the convergence and performance of selecting low-rank patches is not theoretically guaranteed as it may overestimate the noise level for small noise level cases and underestimate the noise level in large noise level cases.

\subsection{Existing Denoising Techniques}

Bilateral filter [8] is introduced for gray and color image processing. The Bilateral filter mixes the color and gray level on both their geometric closeness and their photometric similarity and near values to distant values in both domain and range is preferred. Bilateral filter can enforce the perceptual metric underlying the CIE-Lab color space, and smooth colors and preserve edges which are compatible with human perception. Bilateral filter produces no phantom colors along edges in color images, and reduces phantom colors in the original image. The method is non-iterative, local, and simple. The Bilateral filter considers geometric and photometric distance of candidate pixel in local window. Hence, it removes noise and also retaining edges due to the framework. The Bilateral filter may not identify the different frequency components of a signal. The Bilateral filter is effective in removing high-frequency noise but unable to remove low-frequency noise.

A novel image denoising strategy based on an enhanced sparse representation in transform domain namely Sparse 3D Transformdomain Collaborative filter is also being introduced [9]. The sparsity enhancement is carried out by making a group of similar 2D image fragments to form 3D data arrays. These 3D groups underwent special procedure called collaborative filter. It is achieved through three consecutive steps: 3D group transformation, transform spectrum shrinkage, and inverse 3D transformation. The result will be a $3 \mathrm{D}$ estimate consisting of the jointly filtered grouped image blocks. It is a fast and effective algorithm but blocks that are not similar are removed from the matrix group when the image is in high noise level.

A new model based on the hybridization of Wavelet and Bilateral filters for denoising of variety of noisy images is introduced in [10]. The algorithm is carried out on images such as x-ray, ultrasound and astronomical telescopic images and the performances are analysed based on peak signal to noise ratio (PSNR) and image quality index (IQI) [10]. The filter developed with Bilateral filter before and after image decomposition is found to produce the best denoising performance in terms of PSNR and IQI amongst four models involved in this study. However, the computational time is longer and not very effective in reducing Salt and Pepper noise.

A general method for image contrast enhancement and noise reduction [11] is developed specifically for image enhancement under very low light conditions where the details of images are not so visible and the noise level is high. The intensity of an image can be amplified to make the dark areas become bright and enhancing the contrast by applying an improved and effective image de-haze algorithm to the inverted input image Then, the jointBilateral filter with the original green component as the edge image is implemented to reduce the noise. Based on the results obtained, the proposed method performed much better than some current methods in visual quality. However, the computational time is long for real-time video.

\section{Methodology}

The noise estimation denoising technique developed by combining Patch-based Noise Level Estimator for noise estimation with the OTSU WIE-WATH Filter [4] and OTSU KU-WIE-WATH Filter [4] to eliminate the Poisson noise effectively in real application. The noise level of input image will be estimated by using Patchbased Noise Level Estimator method. The denoising will be performed based on the estimated noise. The estimated noise level which value is less than 0.01 is the image with low and medium Poisson noise. The value 0.01 is the lower boundary of high Poisson noise value that determine by the previous study [4]. Hence, the value of 0.01 is used to differentiate the images into low and medium Poisson noise and high Poisson noise in this research. The flowchart for the proposed method is shown in Figure 1.

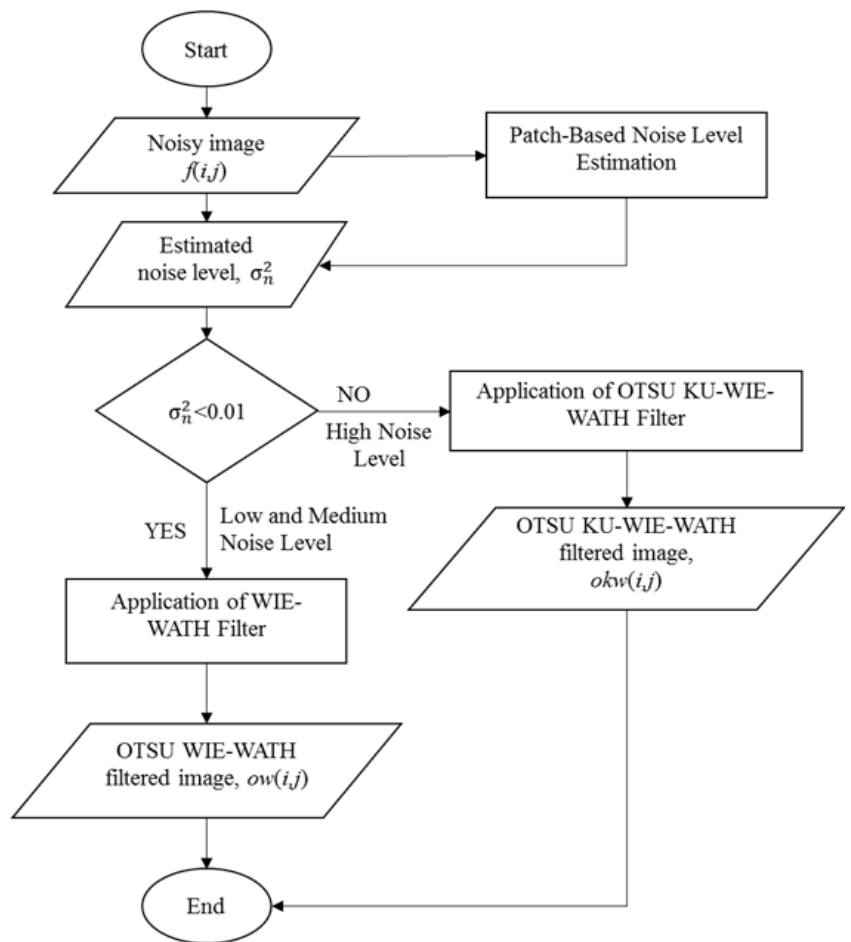

Fig. 1: Flowchart of the proposed method 


\subsection{Patch-Based Noise Level Estimation Method: Weak textured patch selection}

The noisy $\mathrm{f}$ image, $f(i, j)$ can be measured efficiently by using the gradient covariance matrix. Assuming that we have an image patch, $y(p, q)$, from noisy $f$ image, $f(i, j)$. The flowchart for the Patch-Based Noise Level Estimation method is shown in Figure 2.

The level of the Poisson noise, $\lambda$, variance of the Gaussian noise, $\sigma^{2}$, can be adapted to the pixel values by using the following relation:

Poisson $(\lambda)=$ Gaussian $\left(\mu=\lambda, \sigma^{2}=\lambda\right)$

If there is a sufficient large value of Poisson noise, $\lambda$, the normal Gaussian distribution with value of $\lambda$, is an excellent approximation to the Poisson distribution. Hence, obtaining the variance value of Gaussian noise in certain patch enables the estimation of Poisson noise in the corresponding area as well.

\subsection{Iterative framework of noise level estimation}

In patch-based noise level estimation method [7], the input image is categories into several patches in a raster scan. To analyse the structure of image and to select suitable patches from the noisy image, local variance of image patch is widely used. The iterative noise level estimation process is presented in Figure 2.

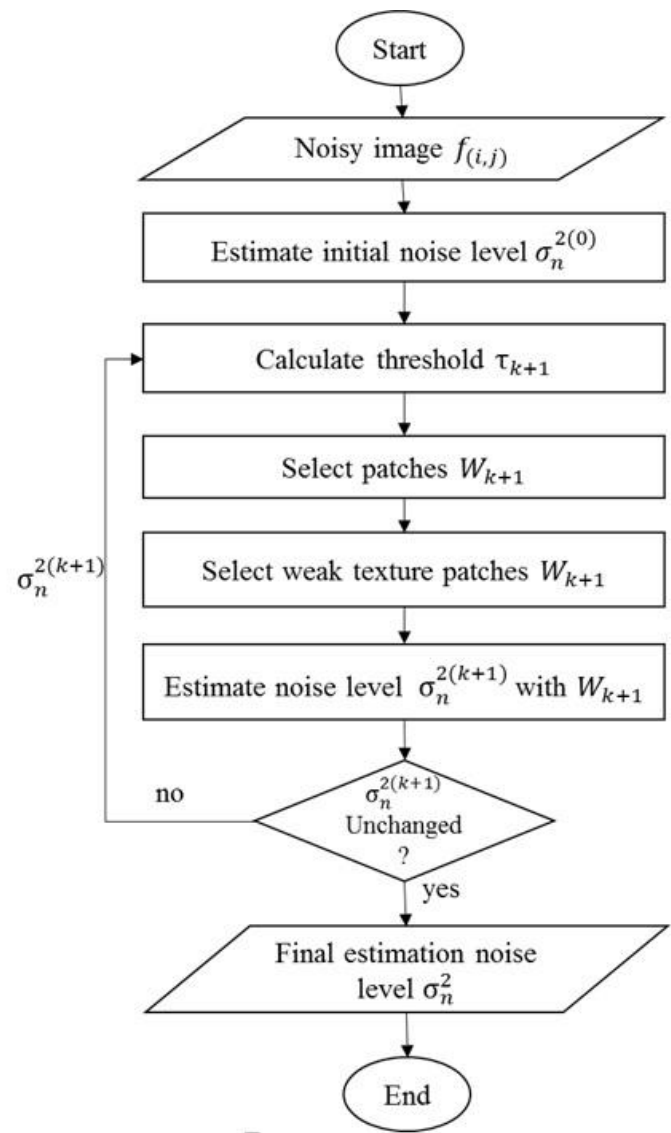

Fig. 2: Flowchart of Patch-based Noise Level Estimation method [7]

First, an initial noise level, $\sigma_{n}^{2(0)}$ is estimated from the covariance matrix, $C_{(y(p, q)}$, which is generated using all patches in the input noisy image. Based on the $\mathrm{k}$-th (the number of iterative) estimated noise level, $\sigma_{n}{ }^{2(k)}$, the $(k+1)$ th threshold $\tau_{(k+1)}$ is determined. The weak textured patch dataset, $W_{(k+1)}$, is selected from the noisy image using the threshold $\tau_{(k+1)}$. Then the $(k+1)$ th noise level, $\sigma_{n}{ }^{2(k+1)}$ is estimated using selected $W_{(k+1)}$ with the threshold $\tau_{(k+1)}$. This process is iterated until the estimated noise level $\sigma_{n}^{2}$ is unchanged.

\subsection{OTSU WIE-WATH Filter}

OTSU WIE-WATH Filter [4] is the combination of Otsu Threshold, Wavelet Threshold and Wiener Filter. This method is well to reduce noise in smooth region of noisy image while preserving the texture region of noisy image. Firstly, the noisy low light image, $f(i, j)$ will be inserted and Otsu Threshold is applied to the noisy image to produce an Otsu binary image $o b w(i, j)$. The level 1 of Otsu binary image represents texture regions and level 0 represents smooth regions. Then WIE-WATH Filter will be applied on the smooth regions of noisy image, $f(i, j)$ based on the location of level 0 in the Otsu binary image $o b w(i, j)$.

Besides, the pivel value of texture region noisy image, $f(i, j)$ based on the location of level 1 in the Otsu binary image $o b w(i, j)$ will be used directly without application of WIE-WATH Filter. Finally, the OTSU WIE-WATH image, ow $(i, j)$ is produced by the combination of noisy image, $f(i, j)$ and the WIE-WATH filtered image, $w(i, j)$ The process flowchart of this proposed OTSU WIE-WATH Filter is shown in Figure 3

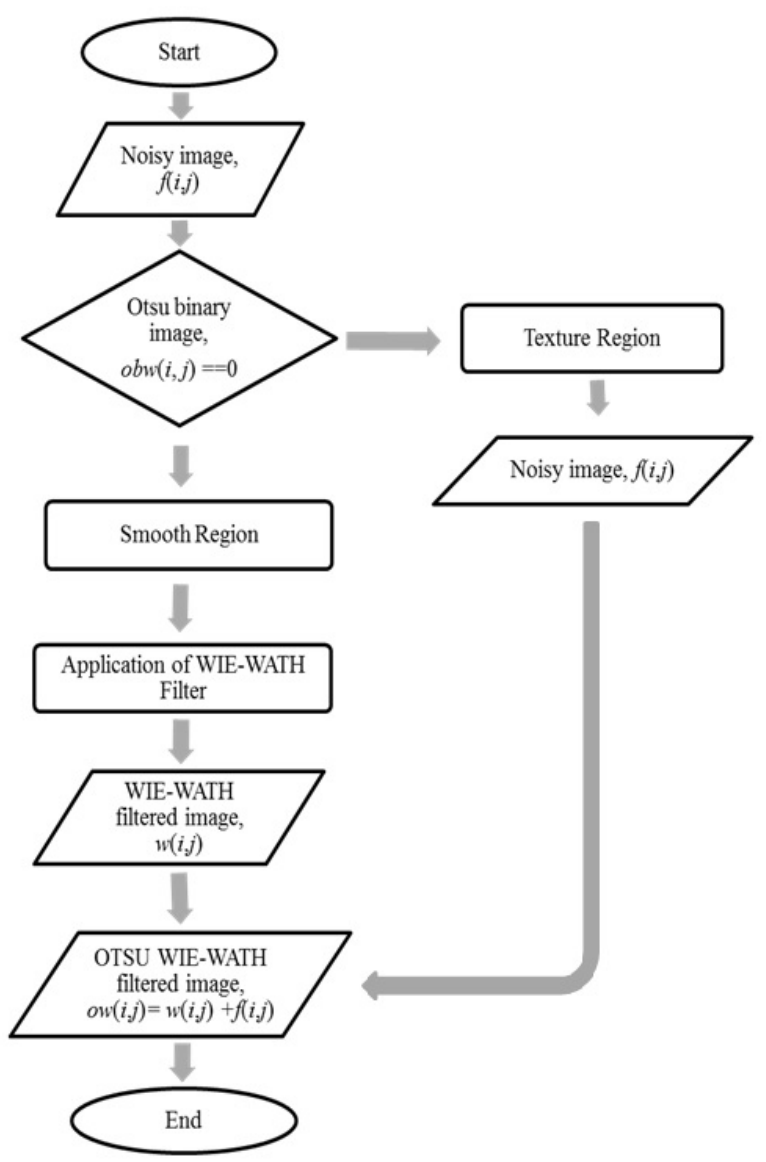

Fig. 3: Flowchart of OTSU WIE-WATH Filter [4]

\subsection{OTSU KU-WIE-WATH Filter}

OTSU KU-WIE-WATH Filter [4] is developed through the combination of Otsu Threshold, Kuwahara Filter, Wavelet Threshold and Wiener Filter. This proposed method will be able to reduce noise in smooth and texture regions of high level noise image. The first step of this method is performed by inserting the noisy low light image, $f(i, j)$. and Otsu Threshold is then applied to the noisy image to produce an Otsu binary image $o b w(i, j)$. The Otsu binary image is made up of two levels which are level 1 and level 0 rep- 
resenting texture and smooth regions respectively. Next, Kuwahara Filter followed by WIE-WATH Filter is applied on the smooth regions of the noisy image, $f(i, j)$ based on level 0 location in the Otsu binary image $o b w(i, j)$ to form KU-WIE-WATH image,

$k w(i, j)$

On the other hand, WIE-WATH Filter will be applied on the texture regions based on the level 1 location in the Otsu binary image $o b w(i, j)$ to form WIE-WATH image, $w(i, j)$. Finally, the OTSU KU-WIE-WATH image, $o k w(i, j)$ produced is the combination of KU-WIE-WATH filtered image, $k w(i, j)$ and WIE-WATH filtered image, $w(i, j)$. The flowchart of this proposed OTSU KU-WIEWATH Filter is shown in Figure 4.

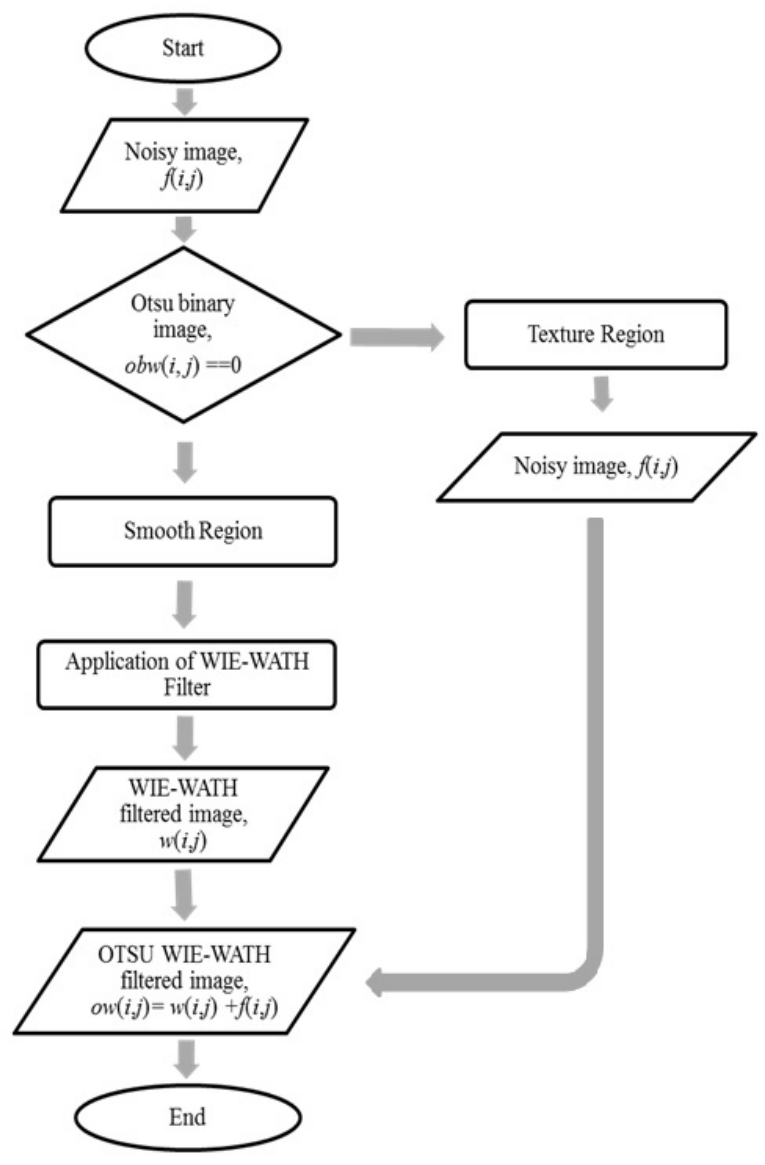

Fig. 4: Flowchart of OTSU KU-WIE-WATH Filter [4]

\section{Results and Analysis}

The outcome and analysis of different noise estimator and denoising filter from testing image by using MATLAB software. This study was carried out to measure the accuracy and effectiveness of the proposed method to remove the low, medium and high level of Poisson noise for low light condition in surveillance image.

To develop an effective denoising technique with noise estimator for Poisson noise removal in low-light condition surveillance image, the noise estimation denoising technique by combining Patchbased Noise Level Estimation method and OTSU WIE-WATH Filter and OTSU KU-WIE-WATH Filter to eliminate the Poisson noise automatically in real application is developed. Proposed method will automatically fed the image with low and medium Poisson noise to the OTSU WIE-WATH Filter while the image with high Poisson noise will be fed to the OTSU KU-WIE-WATH Filter respectively. The performance of the proposed filter will be evaluated through visual effects and computational time.

\subsection{Visual Effect for Low and Medium Noise Removal}

The visual effects inspection is based on the perception of human eye-sight. Hence, a survey form is distributed to 40 student respondents to evaluate the performance of each filter. This evaluation is carried out to find the most effective method in denoising low and medium noisy image. The physical characteristic of the images such as edges, fine details and quantity of noises in processed test images are observed. There are seven images obtained by processing a single test image namely Noisy Image, Proposed Filtered Image, OTSU KU-WIE-WATH Filtered Image, KUWATH Filtered Image, OTSUHARA Filtered Image, Bilateral Filtered Image and the Bilateral+ Wavelet Threshold Filtered Image. The images are the footage obtained from CCTV without the original clear images are utilized in analysis of visual effects. The red square box indicates the texture region while the yellow square box indicates the smooth region.
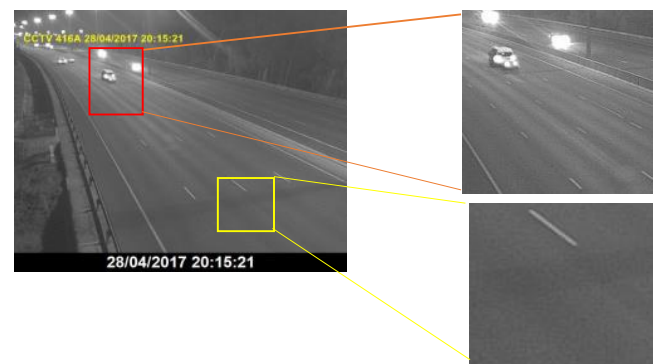

(a) Noisy Image (yellow box: smooth region, red box: texture region) (Estimation noise level, $\lambda: 0.0087$ )

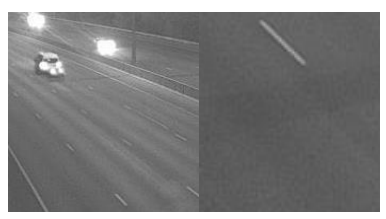

(b) Proposed Filter

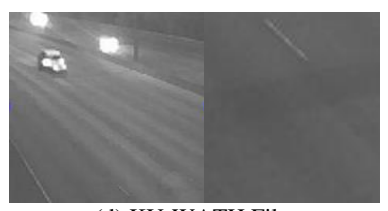

(d) KU-WATH Filter

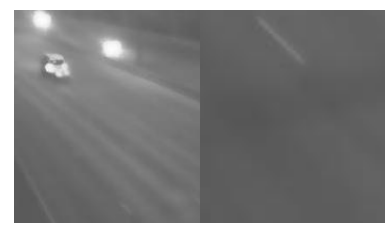

(f) Bilateral Filter

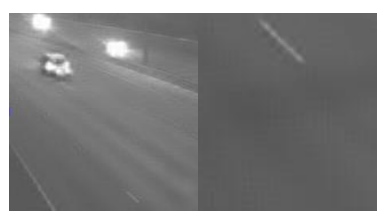

(c) OTSU KU-WIE-WATH Filter

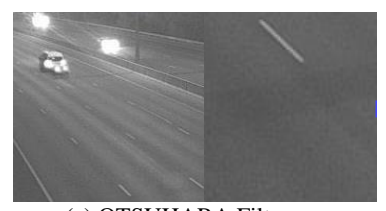

(e) OTSUHARA Filter

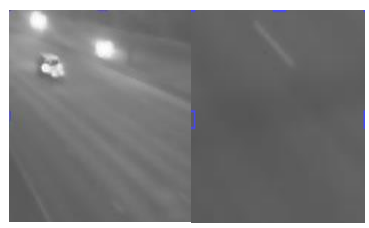

(g) Bilateral + Wavelet Filter
Fig. 5: Visual effects for denoising cctv13.jpg corrupted by low and medium Poisson Noise ((b)-(g) left: enlarged textured region, right: enlarged smooth region).

From Figure 5, it can be observed that Proposed filtered image looks similar to the original image after all noisy pixels in the smooth regions are removed and fine edges and details are wellpreserved in the texture region. OTSUHARA filtered image also has well-preserved texture region but there are some noisy pixels in the smooth region that is not removed thoroughly. OTSU KUWIE-WATH and KU-WATH filtered images have over-smoothed texture regions. The over-smoothing effect is more critical in texture region of Bilateral and Bilateral+Wavelet filtered images which give a blurred visual effect. Thus, it can be proven that the OTSU WIE-WATH Filter has the best visual effects; it is able to remove noises while edges and fine details are preserved in low and medium noise levels images. 
In Figure 6, the result shows for image cctv13.jpg in Figure 5 Proposed Filter has been chosen by $77 \%$, followed by OTSU KUWIE-WATH Filter $20 \%$ and Bilateral Filter $3 \%$.

\subsection{Visual Effect for High Noise Removal}

This section also will observe the image characteristic such as edges, fine details and quantity of noises. The visual effects are based on the perception of human eye-sight. 40 respondents have done a survey form based on their observation. There are seven images obtained by processing a single test image namely Noisy Image, OTSU WIE-WATH Filtered Image, Proposed Filtered Image, KU-WATH Filtered Image, OTSUHARA Filtered Image, Bilateral Filtered Image and the Bilateral+ Wavelet Threshold Filtered Image.

CCTV13

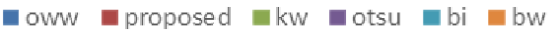

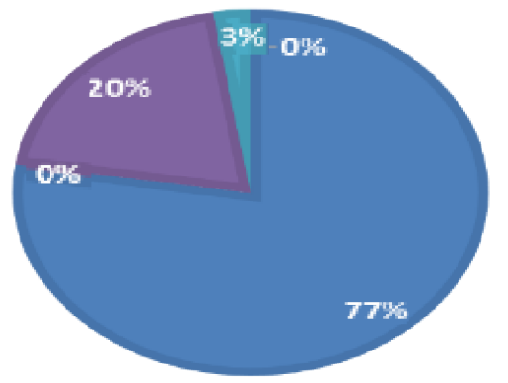

proposed: OTSU WIE-WATH Filter okww: OTSU KU-WIE-WATH Filter kw: KU-WATH Filter otsu: OTSUHARA Filter bi: Bilateral Filter bw: Bilateral + Wavelet Filter

Fig. 6: Analysis of respondents in cctv13.jpg
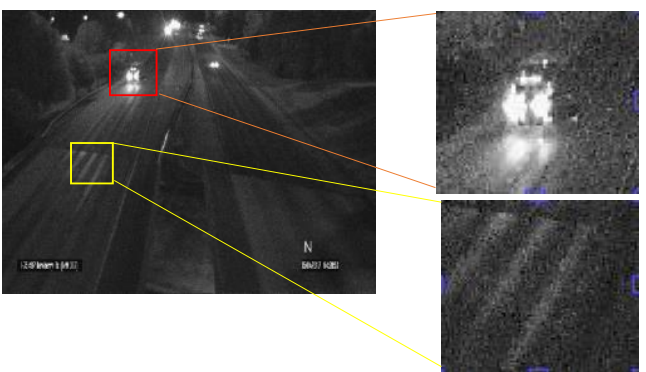

(a) Noisy Image (yellow box: smooth region, red box: texture region) (Estimation noise level, $\lambda$ : 0.0175)

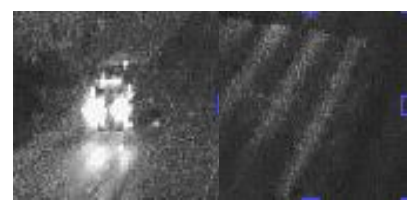

(b) OTSU WIE-WATH Filter

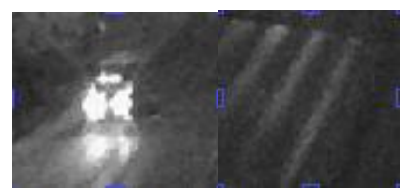

(d) KU-WATH Filter

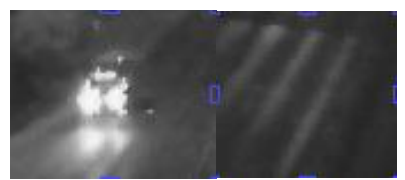

(f) Bilateral Filter

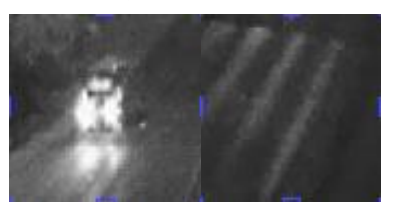

(c) Proposed Filter

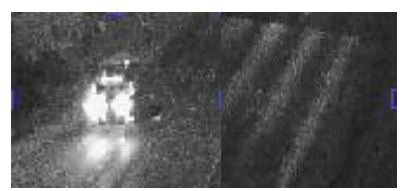

(e) OTSUHARA Filter

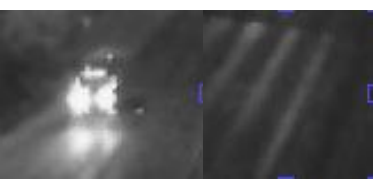

(g) Bilateral + Wavelet Filter
Fig. 7: Visual effects for denoising cctv3.jpg corrupted by high Poisson Noise ((b)-(g) left: enlarged textured region, right: enlarged smooth region)
The images are the footage obtained from CCTV without the original clear images are utilized in analysis of visual effect inspection. The square box indicated the texture region while the round box indicated the smooth region.

From Figure 7, it can be observed that Proposed filtered image preserve image details and texture regions, while removing most af the noise in smooth regions. The OTSU WIE-WATH and OTSUHARA filtered image also has well preserved texture region but there are more noisy pixels in smooth region and has the tendency of pixilation. The KU-WATH filtered images left most noisy pixels in texture regions compared to the Proposed filtered image. The over-smoothing effect exist in high noise level Bilateral and Bilateral+Wavelet filtered images which give a blurred visual effect. Thus, it can be proven that Proposed Filter has better visual effects; it provides well noise removal and preservation of edges and fine details in high noise levels.

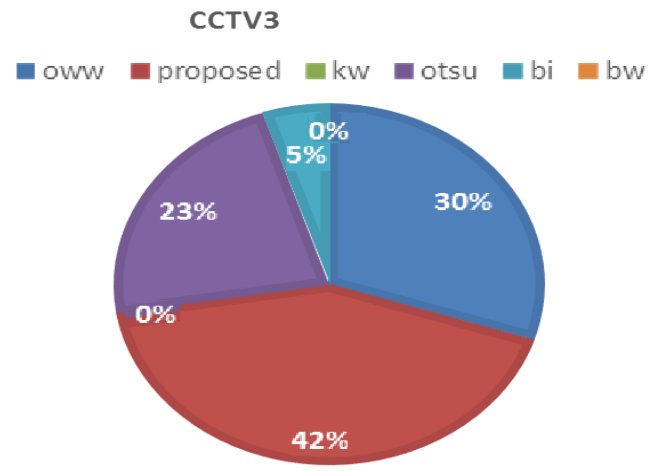

* oww: OTSU WIE-WATH Filter proposed: OTSU KU-WIE-WATH Filter kw: KU-WATH Filter otsu: OTSUHARA Filter bi: Bilateral Filter bw: Bilateral + Wavelet Filter

Fig. 8: Analysis of respondents in cctv3.jpg

In Figure 8, the result shows for image cctv3.jpg in Figure 6, Proposed Filter has been chosen by $42 \%$, followed by OTSU WIEWATH Filter 30\%, OTSUHARA Filter 23\% and Bilateral filter $5 \%$.

\section{Conclusion}

An effective denoising technique for Poisson noise removal in low light condition surveillance images is developed. This project proposed a Patch-based Noise Level Estimator with two denoising techniques OTSU WIE-WATH Filter and OTSU KU-WIEWATH Filter. The Patch-based Noise Level Estimator will categorize the noisy image with the noise level $<0.01$ to OTSU WIEWATH Filter that is specially designed for effective low and medium Poisson noise removal and noisy image with the noise level $\geq 0.01$ to OTSU KU-WIE-WATH Filter that specially designed for effective high Poisson noise removal. The proposed method is able to work automatically in differentiate the noise level of noisy image and remove the low, medium and high Poisson noise effectively.

\section{Acknowledgement}

The authors would like to express gratitude to Universiti Tun Hussein Onn Malaysia for the support of this research.

\section{References}

[1] Gonzalez RC \& Woods RE, Digital Image Processing, Pearson, (2008).

[2] Wueller D, "Low Light Performance of Digital Still Cameras," SPIE :Multimedia Content and Mobile Devices, Vol. 8667, (2013), 
https://spie.org/Publications/Proceedings/Paper/10.1117 $/ 12.2003080$

[3] Kaur S, "Noise types and various removal techniques," International Journal of Advanced Research in Electronics and Communication Engineering Vol. 4, No. 2,( 2015) pp. 226-230, available online: http://ijarece.org/wp-content/uploads/2015/02/ IJARECEVOL-4-ISSUE-2-226-230.pdf, last visit:13.08.2018

[4] Sari S, Chia YY, Mohd MNH, Taujuddin NSAM, Ibrahim N, and Roslan H, "OKWW Filter for Poisson Noise Removal in Low-Light Condition Digital Image," IEEE International Conference on Signal and Image Processing Applications, (2017) pp. 335-340, https://ieeexplore.ieee.org/document/8120632/

[5] Immerkaer J, "Fast Noise Variance Estimation," Computer Vision and Image Understanding, Vol. 64, No. 2, (1996), pp. 300-302, available online: https://www.sciencedirect.com/ science/article/pii/S1077314296900600, last visit: 13.08.2018

[6] Shin DH, Park RH, Yang S \& Jung JH, "Block-based Noise Estimation using Adaptive Gaussian Filtering," IEEE Transactions on Consumer Electronics, Vol. 51, No. 1, (2005), pp. 218-226, available online: https://ieeexplore.ieee.org/document/ 1405723/, last visit: 13.08 .2018

[7] Liu X, Tanaka M, \& Okutomi M, "Single-image Noise Level Estimation for Blind Denoising," IEEE Transactions on Image Processing, Vol. 22, No. 12, (2013), pp. 5226-5237, available online: https://ieeexplore.ieee.org/document/6607209/, last visit: 13.08.2018

[8] Tomasi C \& Manduchi R, "Bilateral Filtering for Gray and Color Images," IEEE International Conference of Computer Vision, (1998), pp. 839-846, https://ieeexplore.ieee.org/ document/ 710815/

[9] Foi A, Trimeche M, Katkovnik V \& Egiazarian K, "Practical Poissonian- Gaussian Noise Modeling and Fitting for Single-Image Raw-Data," IEEE Transactions on Image Processing, Vol. 17, No. 10,( 2008), pp.1737-1754, available online: https://ieeexplore.ieee.org/stamp/stamp.jsp?arnumber=4623175, last visit: 13.08 .2018

[10] Roy S, Sinha N \& Sen AK, "A New Hybrid Image Denoising Method," International Journal of Information Technology and Knowledge Management, Vol. 2, No. 2, (2010), pp. 491-497, http://csjournals.com/IJITKM/PDF\%203-1/63.pdf

[11] Zhang X, Shen P, Luo L, Zhang L \& Song J, "Enhancement and Noise Reduction of Very Low Light Level Images," IEEE International Conference on Pattern Recognition, (2012) pp. 2034-2037, https://ieeexplore.ieee.org/stamp/stamp.jsp?arnum ber $=6460559$ 